\title{
Open Access at Nature Metabolism
}

\section{One year ago, we introduced open access publishing at Nature Metabolism. Here, we review the publishing options} for authors and our progress so far.

tos

he future of science is open and the publishing landscape is changing as a result. The transition from subscription to open access (OA) publishing is irreversible. The benefits of OA for authors are obvious: by making the final version of a research article - the version of record - free to read and discoverable for everyone, OA allows researchers' work to reach a broader audience and to make a bigger impact.

Since January 2021, all authors of newly submitted manuscripts can benefit from these advantages, as they can now choose an OA publishing option when their work is accepted for publication in Nature Metabolism. OA publication is supported by payment of an article processing charge of $€ 9500$, which is typically paid by the authors' institutions and funding bodies, or is covered by 'transformative agreements' (more on these below).

The decision of which publishing option to choose rests solely with the authors (provided they comply with rules set by their funding bodies) and is taken only after formal acceptance of the manuscript. Therefore, the publishing model chosen by authors neither influences editors in their decisions, nor alters the editorial criteria of the journal.

Notably, authors can continue to choose between OA publishing and the traditional subscription publishing model. The subscription model remains free of cost for authors, but makes articles available only to subscribers of the journal (although the accepted version of the manuscript can be deposited in a public or institutional repository after an initial embargo period of six months). It is important to offer authors a choice between OA and the subscription model while different regions of the world are transitioning to OA publishing at varying speeds.

Transformative agreements will probably play a key role in the transition to full OA publishing. The result of complex negotiations between publishers and science funders, institutions or national consortia, such agreements have considerable advantages for authors, including offsetting the cost associated with publishing in OA journals. For example, Springer Nature has a transformative agreement with Max Planck Digital Library, enabling all authors at Max Planck Institutes to publish their work via OA in Nature portfolio journals - including Nature Metabolism - at no direct cost to authors.

So how big a role has OA played at Nature Metabolism so far? Considering that only authors of manuscripts submitted since the beginning of 2021 can choose the OA publishing route and that manuscripts take time to move through the review and revision process, it is fair to say that it is still early days for OA publishing in our journal. In 2021, we published a total of six OA research papers, four of which were published late in the year as part of our December issue. As more papers are advancing through our publication pipeline, we expect to see a steady stream of OA articles appear in our pages, although the exact number will depend on author uptake. As a transformative journal, we remain committed to increasing the share of OA research articles, with the goal of becoming a full OA journal in due course.

Of note, we continue to make COVID19-related research free to read in the context of the ongoing pandemic, even if papers are published under the subscription route (these papers are not counted as OA in this analysis).

While we are keen to continue our transition to OA publishing, our commitment to open research does not stop here. Enhanced reporting and sharing of data, code and results (including in the form of preprints), or insight into the peer review process, all open up science in various ways. As a result, we have plans to further develop and improve the ways in which we support open research across the Nature portfolio and in Nature Metabolism specifically. If you have any suggestions of how we could do better in the process, please do not hesitate to reach out to us - we always welcome your feedback.

Published online: 13 January 2022 https://doi.org/10.1038/s42255-022-00526-8 